\title{
A Review of the Composition of the Essential Oils and Biological Activities of Angelica Species
}

\author{
Kandhasamy Sowndhararajan ${ }^{1}$, Ponnuvel Deepa ${ }^{1}$, Minju Kim ${ }^{1}$, Se Jin Park ${ }^{1}$ and \\ Songmun $\operatorname{Kim}^{1,2, * \text { (iD }}$ \\ 1 School of Natural Resources and Environmental Sciences, Kangwon National University, \\ Chuncheon 24341, Korea; sowndhar1982@gmail.com (K.S.); taanishadeepa@gmail.com (P.D.); \\ camin1121@gmail.com (M.K.); sejinpark@kangwon.ac.kr (S.J.P.) \\ 2 Gangwon Perfume Alchemy Ltd., Co., Chuncheon 24341, Gangwon-do, Korea \\ * Correspondence: perfume@kangwon.ac.kr; Tel.: +82-33-250-6447; Fax: +82-33-241-6440
}

Academic Editor: Gernot A. Eller

Received: 1 September 2017; Accepted: 15 September 2017; Published: 20 September 2017

\begin{abstract}
A number of Angelica species have been used in traditional systems of medicine to treat many ailments. Especially, essential oils (EOs) from the Angelica species have been used for the treatment of various health problems, including malaria, gynecological diseases, fever, anemia, and arthritis. EOs are complex mixtures of low molecular weight compounds, especially terpenoids and their oxygenated compounds. These components deliver specific fragrance and biological properties to essential oils. In this review, we summarized the chemical composition and biological activities of EOs from different species of Angelica. For this purpose, a literature search was carried out to obtain information about the EOs of Angelica species and their bioactivities from electronic databases such as PubMed, Science Direct, Wiley, Springer, ACS, Google, and other journal publications. There has been a lot of variation in the EO composition among different Angelica species. EOs from Angelica species were reported for different kinds of biological activities, such as antioxidant, anti-inflammatory, antimicrobial, immunotoxic, and insecticidal activities. The present review is an attempt to consolidate the available data for different Angelica species on the basis of major constituents in the EOs and their biological activities.
\end{abstract}

Keywords: Angelica; bioactivity; essential oil; hydrodistillation; steam distillation

\section{Introduction}

In traditional systems of medicine, a number of plants have been widely used for the treatment of various disorders since ancient times. Plants are a versatile source of bioactive metabolites, including polysaccharides, phenolics, alkaloids, essential oils (EOs), steroids, lignins, resins, tannins, etc. [1]. Among them, EOs obtained from plants have various applications, especially in the health, agriculture, food, and cosmetic industries. So far, more than 3000 EOs have been isolated from about 2000 plant species, out of which 300 have been commercially used for various purposes [2]. Previous scientific studies clearly revealed that EOs possess various pharmacological properties such as antioxidant, antimicrobial, antiviral, antimutagenic, anticancer, anti-inflammatory, and immunomodulatory activities [3].

EOs are mainly stored in the oil ducts, resin ducts, glands, or trichomes of the plants [2]. They are a complex mixture of low molecular weight volatile compounds, mainly monoterpenes and sesquiterpenes, and their oxygenated derivatives. Each type of EO contains about 20-100 different components from a variety of chemical classes [4]. In general, the bioactivities of a particular EO are decided by its major components [3]. However, the presence of minor components also plays an essential role in the bioactivities of EOs. They can be obtained from different organs of various medicinal and aromatic plant materials using classical and advanced techniques. Hydrodistillation and steam distillation 
are the most important conventional techniques to isolate EOs. The gas chromatography and mass spectrometry (GC-MS) is a widely used method to determine the chemical composition of EOs [5,6]. The compositions of EOs are highly influenced by various parameters, such as harvesting season, plant organs, plant maturity, genetic diversity, nutritional status, environmental conditions, drying methods, and extraction and analysis techniques $[7,8]$.

The genus Angelica L. belongs to the family of Apiaceae (Umbelliferae), comprises about 90 species of biennial perennial herbs that are widely distributed in Asia, Europe, and North America. In these, a total of 45 Angelica species (32 endemic species) are distributed in China [9]. Various species of Angelica have been used in the traditional systems of medicine for several centuries. Previously, several authors reported the volatile composition of different Angelica species using various extraction techniques such as steam distillation, hydrodistillation, solvent-free solid injector, and supercritical fluid extraction. EOs of Angelica species exhibit several pharmacological activities, such as antioxidant, antibacterial, antifungal, antimicrobial, and insecticidal activities. The present paper summarizes the compositions and biological activities of EOs from different Angelica species (Tables 1 and 2).

\section{Traditional Uses of Angelica Species}

Traditionally, Angelica sinensis, Angelica gigas, and Angelica acutiloba are the most important Angelica species, which are mainly found in Korea, China, and Japan, respectively. In China, A. sinensis has been used for the treatment of various ailments such as gynecological diseases, apoplexia, constipation, malaria, chills, fever, and hemorrhoids. The plant has also been used as a hematinic for nourishing blood, regulating menstruation, and relaxing bowels [10-12]. In the Korean traditional medicine, the root part of $A$. gigas has been to treat anemia, gynecological diseases, circulatory diseases, and arthritis. It has also been used as sedative, analgesic, and tonic agents $[13,14]$. A. acutiloba is traditionally used to treat gynecological diseases and anemia [15]. Angelica archangelica is commonly used in traditional medicine to cure nervousness, insomnia, stomach and intestinal disturbances, skin diseases, respiratory problems, and arthritis [16,17]. Angelica glauca has been used to treat bilious complaints, infantile atrophy, and constipation [18]. Angelica dahurica has been mainly used to treat headaches, rhinitis, toothaches, rheumatism, and sore throat [19]. Angelica pubescentis has been used to cure rheumatoid arthritis, headache, paralysis, and insomnia [20].

\section{The Chemical Composition of Essential Oils of Angelica Species}

The main aim of this review is to offer an overview on the chemical composition of EOs from different species of Angelica growing in various countries. Table 1 shows the plant name, plant parts, extraction methods, yield, and the major components of EOs in relation to different species of Angelica. The published reports revealed that the EOs of the genus Angelica isolated by steam distillation or the hydrodistillation method mainly consist of monoterpene hydrocarbons. Figure 1 depicts the chemical structure of some of the major components of EOs from Angelica species.

In $A$. archangelica seed EOs, $\beta$-phellandrene (33.6-63.4\%) and $\alpha$-pinene (4.2-12.8\%) were detected as the most abundant components [21]. On the other hand, $\alpha$-pinene $(21.3 \%), \delta$-3-carene $(16.5 \%)$, limonene $(16.4 \%)$, and $\alpha$-phellandrene $(8.7 \%)$ were the most abundant components in the EO of A. archangelica roots growing in Italy [22]. Nivinskiene et al. [23] studied the EO composition of A. archangelica roots collected from three habitats (Svencionys, Prienai, and Vilnius districts in Lithuania) between 1995-2002. $\alpha$-Pinene (15.7-20.8\%) was the major EO component in two localities, whereas $\beta$-phellandrene (13.8-18.5\%) and $\alpha$-pinene (11.4-15.0\%) were registered as the major EO components in the third locality. The EOs contained $67.3-79.9 \%$ of monoterpenes, $9.6-19.4 \%$ of sesquiterpenes, and 3.9-6.3\% of macrocyclic lactones. Chauhan et al. [17] found that the EOs of A. archangelica rhizomes obtained from three different altitudes of western Himalaya mainly contained dillapiole (35.93-91.55\%) and nothoapiole (0.1-62.8\%). Further, the authors reported that the composition of EOs varied greatly with the altitude of collection. Pasqua et al. [16] investigated the accumulation of EOs in the roots of 
A. archangelica subsp. archangelica at different developmental stages. A high concentration of $\alpha$ - and $\beta$-phellandrene was found only in taproots exceeding $5 \mathrm{~mm}$ in diameter.

The EO of the A. glauca whole plant collected from Jammu and Kashmir mainly contains $\alpha$-phellandrene $(18.0 \%)$, trans-carveol $(16.4 \%), \beta$-pinene $(14.0 \%), \beta$-caryophyllene $(8.6 \%)$, and $\beta$-caryophyllene oxide (8.0\%) [24]. Agnihotri et al. [18] investigated the composition of EO from fresh aerial parts of A. glauca growing in Kashmir valley in higher Himalaya (India), and found that $\alpha$-phellandrene $(13.5 \%)$, trans-carveol $(12.0 \%)$, and $\beta$-pinene $(11.7 \%)$ were the major components. The EOs from the roots of $A$. glauca collected from two alpine Himalayan locations in Uttarakhand (India) highly contain (Z)-ligustilide (40.6-53.0\%) and (Z)-butylidene phthalide (20.7-32.8\%) [25].<smiles>CC1=CCC2CC1C2(C)C</smiles>

$\alpha-P i n e n e$<smiles>CC1=CCC2C(C1)C2(C)C</smiles>

$\mathrm{CH}_{3}$<smiles>C=C1CCC2CC1C2(C)C</smiles>

p-Pinene<smiles>C=C1CCC(C(C)C)CC1</smiles>

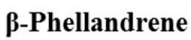<smiles>CC1=CCC(C(C)C)C=C1</smiles>

a-Phellandrene<smiles>C=C1CC[C@@]2(C(C)C)CC[C@@H]1C2</smiles><smiles>CC1=CCC(C(C)C)=CC1</smiles><smiles>C=C(C)[C]1CC=C(C)CC1</smiles>

Limonene<smiles>Cc1ccc(C(C)C)cc1</smiles>

p-Cymene $\gamma$-Terpinene<smiles>CCC/C=C1\OC(=O)C2=C1CCC=C2</smiles><smiles>CCC/C=C1\OC(=O)c2ccccc21</smiles>

Butylidene phthalide<smiles>CC1=C[C@H]2[C@@H](C(C)C)CC[C@](C)(O)[C@@H]2CC1</smiles>

a-Cadinol<smiles>C=C1CCC[C@]2(C)CC[C@H](C(C)(C)O)C[C@@H]12</smiles>

$\beta$-Eudesmol

Figure 1. The chemical structure of some major essential oil components from Angelica species. 
Table 1. The isolation of essential oils and extracts from different Angelica species, and their major components.

\begin{tabular}{|c|c|c|c|c|c|c|}
\hline S. No. & Species & Parts & $\begin{array}{l}\text { Extraction Method; } \\
\text { Extraction Time; Yield }\end{array}$ & Place of Collection & Major Components & References \\
\hline \multirow{6}{*}{1} & \multirow{6}{*}{ Angelica archangelica $\mathrm{L}$. } & $\begin{array}{l}\text { Seeds (fruits) from } \\
\text { three habitats }\end{array}$ & $\begin{array}{l}\text { Hydrodistillation; } 2 \mathrm{~h} ; \\
0.8-1.4 \%\end{array}$ & $\begin{array}{l}\text { Svencionys, Prienai and Vilnius } \\
\text { districts in Lithunia }\end{array}$ & $\begin{array}{l}\beta \text {-phellandrene }(33.6-63.4 \%) \text { and } \alpha \text {-pinene } \\
(4.2-12.8 \%)\end{array}$ & [21] \\
\hline & & Fruit of two chemotypes & $\begin{array}{l}\text { Steam distillation; } 5 \mathrm{~h} ; \\
0.17-0.51 \%\end{array}$ & Reykjavik, Iceland & $\begin{array}{l}\alpha \text {-pinene }(41.4 \%, 28.9 \%, 14.4 \%) \text {, bicyclogermacrene } \\
(10.1 \%) \text {, and } \beta \text {-phellandrene }(37.8 \% \text { and } 55.2 \%)\end{array}$ & [26] \\
\hline & & $\operatorname{Root}(1-2,3-4$ and $>5 \mathrm{~mm})$ & Hydrodistillation; 30 min & Rome, Italy & $\begin{array}{l}\alpha \text {-pinene }(23.89-32.69 \%) \text { and } 8-3 \text {-carene } \\
(3.41-17.07 \%)\end{array}$ & [16] \\
\hline & & Root (3 habitats) & $\begin{array}{l}\text { Hydrodistillation; } 2 \mathrm{~h} ; \\
\quad 0.2-0.5 \%\end{array}$ & $\begin{array}{l}\text { Svencionys, Prienai and Vilnius } \\
\text { districts in Lithuania }\end{array}$ & $\begin{array}{l}\alpha \text {-pinene }(15.7-20.8 \%), \delta-3 \text {-carene }(15.4-16.9 \%), \\
\text { limonene }(8.0-9.2 \%), \beta \text {-phellandrene }(13.5-15.4 \%), \\
\alpha \text {-phellandrene }(8.0-9.1 \%) \text {, and } p \text {-cymene } \\
(6.8-10.6 \%)\end{array}$ & [23] \\
\hline & & Root (3 different altitudes) & $\begin{array}{l}\text { Hydrodistillation; } 3 \mathrm{~h} ; \\
\quad 0.28-0.35 \%\end{array}$ & $\begin{array}{l}\text { Uttarakashi, Rudraprayag and } \\
\text { Pothiwasa in Uttarakhand, India }\end{array}$ & $\begin{array}{l}\text { dillapiole }(35.93-91.55 \%) \text { and nothoapiole } \\
(0.14-62.81 \%)\end{array}$ & [17] \\
\hline & & Root & Hydrodistillation; $2 \mathrm{~h} ; 0.9 \%$ & Urbino, Italy & $\begin{array}{l}\alpha \text {-pinene }(21.3 \%) \text {, , } \delta \text {-3-carene }(16.5 \%) \text {, limonene } \\
(16.4 \%) \text {, and } \alpha \text {-phellandrene }(8.7 \%)\end{array}$ & {$[22,27]$} \\
\hline \multirow{4}{*}{2} & \multirow{4}{*}{$\begin{array}{l}\text { Angelica acutiloba } \\
\text { (Siebold \& Zucc.) Kitag. }\end{array}$} & Leaves, petiole and root & $\begin{array}{l}\text { Hydrodistillation; } 3 \mathrm{~h} ; \\
0.44 \%\end{array}$ & $\begin{array}{l}\text { Rutgers University, New } \\
\text { Brunswick, NJ, USA }\end{array}$ & $\begin{array}{l}\text { Leaves: ligustilide }(11.61 \%) \text { and butylidene } \\
\text { phthalide }(7.29 \%) \\
\text { Petiole: butylidene phthalide }(10.76 \%) \\
\text { Root: nonane }(24.85 \%) \text { and } \alpha \text {-pinene }(31.59 \%)\end{array}$ & [28] \\
\hline & & Root & $\begin{array}{l}\text { Solvent free solid injector; } \\
\text { injection time- } 5 \text { min and } \\
\text { pre-heating time- } 7 \mathrm{~min} \text { ) }\end{array}$ & $\begin{array}{l}\text { Yeosu Province, Republic of } \\
\text { Korea }\end{array}$ & $\begin{array}{l}\text { butylidene phthalide }(17.82 \%) \text {, furfural }(13.67 \%) \text {, } \\
\text { 2-furanmethanol }(11.97 \%), 5 \text {-methyl furfural } \\
(8.50 \%) \text {, maltol }(7.28 \%) \text {, and butylidene } \\
\text { dihydro-phthalide }(5.78 \%)\end{array}$ & [29] \\
\hline & & \multirow{2}{*}{ Root, stem and leaves } & $\begin{array}{c}\text { Steam distillation; } 5 \mathrm{~h} ; 0.05 \\
\text { (root), } 0.06 \text { (stem), and } \\
0.12 \text { (leaves) } \\
\end{array}$ & Nantou, Taiwan & $\begin{array}{l}\text { 3n-butyl phthalide }(30.8-37.9 \%), \gamma \text {-terpinene } \\
(21.1-27.2 \%), p \text {-cymene }(3.6-11.6 \%) \text {, and } \\
\text { cis- } \beta \text {-ocimene }(7.0-7.4 \%)\end{array}$ & [30] \\
\hline & & & $\begin{array}{l}\text { Headspace-solid phase } \\
\text { microextraction; } 20 \text { min }\end{array}$ & Nantou, Taiwan & $\begin{array}{l}\gamma \text {-terpinene }(41.2-52.1 \%), p \text {-cymene }(10.6-17.0 \%), \\
\beta \text {-myrcene }(6.7-8.6 \%), \text {, cis- } \beta \text {-ocimene }(4.9-7.4 \%), \\
\text { and alloocimene }(4.2-5.3 \%)\end{array}$ & [30] \\
\hline \multirow{3}{*}{3} & \multirow{3}{*}{ Angelica glauca Edgew } & Whole plant & $\begin{array}{l}\text { Hydrodistillation; } 3 \mathrm{~h}, \\
\quad 0.17 \%\end{array}$ & Jammu and Kashmir, Pakistan & $\begin{array}{l}\alpha \text {-phellandrene }(18.0 \%), \beta \text {-pinene }(14.0 \%) \text {, } \\
\text { trans-carveol }(16.4 \%), \beta \text {-caryophyllene }(8.6 \%) \text {, and } \\
\beta \text {-caryophyllene oxide }(8.0 \%) \text {. }\end{array}$ & [24] \\
\hline & & Aerial parts & $\begin{array}{l}\text { Hydrodistillation; } 3 \mathrm{~h} ; \\
0.12 \%\end{array}$ & $\begin{array}{l}\text { Khillanmarg areas of Kashmir, } \\
\text { India }\end{array}$ & $\begin{array}{l}\alpha \text {-phellandrene }(13.5 \%) \text {, trans-carveol }(12.0 \%), \\
\beta \text {-pinene }(11.7 \%), \text { thujene }(7.5 \%), \beta \text {-caryophyllene } \\
\text { oxide }(7.2 \%), \beta \text {-caryophyllene }(7.0 \%), \gamma \text {-terpinene } \\
(6.7 \%), \text { nerolidol }(6.5 \%) \text {, and } \beta \text {-bisabolene }(5.2 \%)\end{array}$ & [18] \\
\hline & & Root & $\begin{array}{l}\text { Hydrodistillation; } 5 \mathrm{~h} ; 0.3 \% \\
\text { and } 1.8 \%\end{array}$ & $\begin{array}{l}\text { Himalayan locations of } \\
\text { Uttarakhand, India }\end{array}$ & $\begin{array}{l}\text { (Z)-ligustilide }(40.6-53.0 \%) \text {, (Z)-butylidene } \\
\text { phthalide }(20.7-32.8 \%) \text {, and (E)-butylidene } \\
\text { phthalide }(2.5-5.9 \%)\end{array}$ & [25] \\
\hline
\end{tabular}


Table 1. Cont.

\begin{tabular}{|c|c|c|c|c|c|c|}
\hline S. No. & Species & Parts & $\begin{array}{l}\text { Extraction Method; } \\
\text { Extraction Time; Yield }\end{array}$ & Place of Collection & Major Components & References \\
\hline \multirow{6}{*}{4} & \multirow{6}{*}{ Angelica gigas Nakai } & Leaves, petiole and root & $\begin{array}{l}\text { Hydrodistillation; } 3 \mathrm{~h} ; \\
0.18 \%\end{array}$ & $\begin{array}{l}\text { Rutgers University, New } \\
\text { Brunswick, NJ, USA }\end{array}$ & $\begin{array}{l}\text { Leaves: nonane }(10.75 \%), \alpha \text {-pinene }(33.07 \%) \text {, and } \\
\text { germacrene }(10.05 \%) \\
\text { Petiole: nonane }(8.85 \%), \alpha \text {-pinene }(40.59 \%) \text {, } \\
\beta \text {-phellandrene }(7.52 \%) \text {, and myrcene }(6.38 \%) \text { Root: } \\
\gamma \text {-terpinene }(14.08 \%) \text { and ligustilide }(46.63 \%)\end{array}$ & [28] \\
\hline & & \multirow{5}{*}{ Root } & Hydrodistillation; $4 \mathrm{~h}$ & $\begin{array}{l}\text { Yeosu Province, Republic of } \\
\text { Korea }\end{array}$ & $\begin{array}{l}\text { nonane }(19.99 \%), \alpha \text {-pinene }(44.31 \%) \text {, camphene } \\
(6.66 \%) \text {, and } \delta \text {-limonene }(6.26 \%)\end{array}$ & [29] \\
\hline & & & $\begin{array}{l}\text { Solvent-free solid injector; } \\
\text { injection time- } 5 \mathrm{~min} \text { and } \\
\text { pre-heating time }-7 \mathrm{~min} \text { ) }\end{array}$ & $\begin{array}{l}\text { Yeosu Province, Republic of } \\
\text { Korea }\end{array}$ & $\begin{array}{l}\text { decursin }(29.34 \%) \text {, decursinol angelate }(16.83 \%) \text {, } \\
\text { lomatin }(10.25 \%) \text {, and marmesin }(9.33 \%)\end{array}$ & [29] \\
\hline & & & 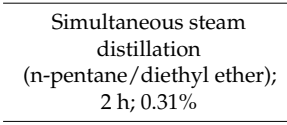 & Gwangju, Republic of Korea & $\begin{array}{l}\alpha \text {-pinene }(30.89 \%), 2,4,6 \text {-trimethyl heptane }(13.39 \%) \text {, } \\
\alpha \text {-limonene }(4.29 \%) \text {, and camphene }(4.10 \%)\end{array}$ & [31] \\
\hline & & & $\begin{array}{l}\text { Steam distillation; } 1 \mathrm{~h} 30 \\
\text { min; } 0.31 \%\end{array}$ & Pyeongchang, Republic of Korea & $\begin{array}{l}\alpha \text {-pinene }(28.64 \%), \beta \text {-eudesmol }(14.80 \%) \text {, nonane } \\
(8.49 \%) \text {, and } \gamma \text {-eudesmol }(5.97 \%)\end{array}$ & [14] \\
\hline & & & $\begin{array}{c}\text { Supercritical } \mathrm{CO}_{2} \\
\text { extraction; } 1 \mathrm{~h} ; 1.67 \%\end{array}$ & Pyeongchang, Republic of Korea & $\begin{array}{l}\text { decursin }(40.13 \%) \text {, decursinol angelate }(28.44 \%) \text {, } \\
\text { and } \beta \text {-eudesmol }(7.84 \%)\end{array}$ & [14] \\
\hline \multirow{3}{*}{5} & \multirow{3}{*}{$\begin{array}{l}\text { Angelica sinensis (Oliv.) } \\
\text { Diels }\end{array}$} & \multirow{3}{*}{ Root } & Hydrodistillation; $8 \mathrm{~h} ; 0.3 \%$ & Gansu Province, China & $\begin{array}{l}\text { (Z)-ligustilide } 78.61 \% \text { and (Z)-butylidenephthalide } \\
7.99 \%\end{array}$ & {$[32-34]$} \\
\hline & & & $\begin{array}{l}\text { Solvent free solid injector; } \\
\text { injection time- } 5 \mathrm{~min} \text { and } \\
\text { pre-heating time }-7 \mathrm{~min} \text { ) }\end{array}$ & $\begin{array}{l}\text { Yeosu Province, Republic of } \\
\text { Korea }\end{array}$ & $\begin{array}{l}\text { butylidene dihydro-phthalide, }(15.23 \%) \text {, butylidene } \\
\text { phthalide }(14.27 \%) \text {, furfural }(16 \%) \text {, camphene } \\
(10.66 \%) \text {, and 4-pyridinol }(7.17 \%)\end{array}$ & [29] \\
\hline & & & $\begin{array}{l}\text { Steam distillation; } 3 \mathrm{~h} ; \\
0.02 \%\end{array}$ & Chiang Mai province, Thailand & $\begin{array}{l}\text { 3-N-butylphthalide, butylidenephthalide, } \\
\text { ligustilide and di-iso-octyl phthalate }\end{array}$ & [35] \\
\hline 6 & Angelica koreana Maxim. & Root & Steam distillation; $0.28 \%$ & $\begin{array}{l}\text { Jinbu, Gangwon-do, Republic of } \\
\text { Korea }\end{array}$ & $\begin{array}{l}\text { sabinene }(31.85 \%) \text {, m-cresol }(4.46 \%), \alpha \text {-pinene } \\
(4.00 \%) \text {, and } \alpha \text {-bisabolol }(3.63 \%)\end{array}$ & [36] \\
\hline \multirow[t]{2}{*}{7} & \multirow{2}{*}{$\begin{array}{l}\text { Angelica dahurica (Fisch. } \\
\text { Ex Hoffm.) Benth. \& } \\
\text { Hook. }\end{array}$} & \multirow[t]{2}{*}{ Root } & $\begin{array}{c}\text { Supercritical } \mathrm{CO}_{2} \\
\text { extraction; } 2 \mathrm{~h} ; 1.8 \%\end{array}$ & Jilin, China & $\begin{array}{l}\text { dodecyl alcohol }(13.71 \%) \text {, elemene }(7.54 \%) \text {, } \\
\text { hexadecanoic acid, ethyl ester }(7.32 \%), \\
\text { 1-pentadecanol }(6.08 \%) \text {, and } \alpha \text {-pinene }(6.25 \%) \text {, }\end{array}$ & [19] \\
\hline & & & $\begin{array}{l}\text { Hydrodistillation; } 3 \mathrm{~h} ; \\
0.45 \%\end{array}$ & Beijing, China & $\begin{array}{l}\alpha \text {-pinene }(46.3 \%) \text {, sabinene }(9.3 \%) \text {, myrcene }(5.5 \%) \text {, } \\
\text { 1-dodecanol }(5.2 \%) \text {, and terpinen- } 4 \text {-ol }(4.9 \%) .\end{array}$ & [20] \\
\hline 8 & $\begin{array}{l}\text { Angelica pancicii Vandas } \\
\text { ex Velen. }\end{array}$ & Root & Hydrodistillation; $2 \mathrm{~h}$ & Balkan mountains, Serbia & $\begin{array}{l}\text { Liquid and headspace injection modes: } \\
\beta \text {-phellandrene }(54.9 \% \text { and } 60.1 \%), \alpha \text {-pinene }(14.5 \% \\
\text { and } 20.1 \%) \text {, and } \alpha \text {-phellandrene }(4.5 \% \text { and } 4.3 \%) \text {. }\end{array}$ & {$[37]$} \\
\hline 9 & $\begin{array}{l}\text { Angelica pubescentis } \\
\text { Maxim. }\end{array}$ & Root & $\begin{array}{l}\text { Hydrodistillation; } 3 \mathrm{~h} ; \\
0.65 \%\end{array}$ & Beijing, China & $\begin{array}{l}\alpha \text {-pinene }(37.6 \%), p \text {-cymene }(11.6 \%) \text {, limonene } \\
(8.7 \%) \text {, and cryptone }(6.7 \%)\end{array}$ & [20] \\
\hline 10 & $\begin{array}{l}\text { Angelica urumiensis } \\
\text { (Mozaffarian) }\end{array}$ & Stem & Hydrodistillation; $3 \mathrm{~h} ; 0.2 \%$ & $\begin{array}{l}\text { Uremia, Province West } \\
\text { Azerbaijan, Iran }\end{array}$ & $\begin{array}{l}\text { Stem: } \alpha \text {-cadinol }(9.24 \%),(\text { epi)- } \alpha \text {-cadinol }(5.76 \%) \text {, } \\
\text { and } \delta \text {-cadenine }(6.11 \%)\end{array}$ & {$[38]$} \\
\hline
\end{tabular}


Table 1. Cont.

\begin{tabular}{|c|c|c|c|c|c|c|}
\hline S. No. & Species & Parts & $\begin{array}{l}\text { Extraction Method; } \\
\text { Extraction Time; Yield }\end{array}$ & Place of Collection & Major Components & References \\
\hline 11 & $\begin{array}{l}\text { Angelica urumiensis } \\
\text { (Mozaffarian) }\end{array}$ & Leaves & $\begin{array}{l}\text { Hydrodistillation; } 3 \mathrm{~h} ; \\
0.18 \%\end{array}$ & $\begin{array}{l}\text { Uremia, Province West } \\
\text { Azerbaijan, Iran }\end{array}$ & $\begin{array}{l}\text { Leaves: } \alpha \text {-cadinol }(20.2 \%) \text {, hexahydrofarnesyl } \\
\text { acetone }(10.03 \%), 1-\text { dodecanol }(7.55 \%) \text {, linoleic acid } \\
(6.37 \%) \text { and oleic acid }(5.34 \%)\end{array}$ & [38] \\
\hline 12 & $\begin{array}{c}\text { Angelica viridiflora } \\
\text { (Turcz.) Benth. ex Maxim. }\end{array}$ & Aerial parts & Steam distillation; $2 \mathrm{~h} ; 0.2 \%$ & $\begin{array}{l}\text { Shkotovskii District, Primorsky } \\
\text { Krai, Russia }\end{array}$ & $\begin{array}{l}\text { caryophyllene oxide }(61.7 \%) \text { and } \\
\text { 3,4-dimethyl-3-cyclohexan-1-carboxaldehdye (5.8\%) }\end{array}$ & [39] \\
\hline 13 & Angelica cincta Boissieu & Aerial parts & Steam distillation; $2 \mathrm{~h} ; 0.2 \%$ & $\begin{array}{l}\text { Shkotovskii District, Primorsky } \\
\text { Krai, Russia }\end{array}$ & $\begin{array}{l}\alpha \text {-pinene }(67.2 \%) \text {, sabinene }(5.8 \%) \text { and } \beta \text {-pinene } \\
(4.9 \%)\end{array}$ & [39] \\
\hline
\end{tabular}

Table 2. Biological activities of essential oils from different Angelica species.

\begin{tabular}{|c|c|c|c|c|c|}
\hline S. No. & Species & Parts & Biological activity & Model & References \\
\hline \multirow{5}{*}{1} & \multirow{5}{*}{ Angelica archangelica $\mathrm{L}$. } & Seeds & Antioxidant & $\begin{array}{l}\text { Aldehyde/Carboxylic Acid Assay, DPPH radical } \\
\text { scavenging assay, and Malonaldehyde/Gas } \\
\text { Chromatography Assay }\end{array}$ & [40] \\
\hline & & Fruit of two chemotypes & Cytotoxic effect & $\begin{array}{l}\text { Human pancreas cancer cell line PANC-1 and the mouse } \\
\text { breast cancer cell line Crl }\end{array}$ & [26] \\
\hline & & \multirow{3}{*}{ Root } & Anti-seizure & $\begin{array}{l}\text { Maximal electroshock and pentylenetetrazol-induced } \\
\text { seizures in mice }\end{array}$ & [41] \\
\hline & & & $\begin{array}{l}\text { Anti-aflatoxigenic and } \\
\text { antioxidant activities }\end{array}$ & Aspergillus flavus DPPH radical scavenging assay & [42] \\
\hline & & & Antimicrobial & $\begin{array}{l}\text { Fusarium genus, Botrytis cinerea, and Alternaria solani, } \\
\text { Clostridium difficile, Clostridium perfringens, Enterococcus } \\
\text { faecalis, Eubacterium limosum, Peptostreptococcus anaerobius, } \\
\text { and Candida albicans }\end{array}$ & {$[22,27]$} \\
\hline \multirow{3}{*}{2} & \multirow{3}{*}{ Angelica gigas Nakai } & \multirow{2}{*}{ Root } & $\begin{array}{l}\text { Nicotine } \\
\text { Sensitization }\end{array}$ & $\begin{array}{l}\text { Repeated nicotine-induced locomotor activity and } \\
\text { extracellular dopamine levels in the nucleus accumbens } \\
\text { of rats }\end{array}$ & [43] \\
\hline & & & Human EEG & $\begin{array}{l}\text { Increased absolute low beta (left temporal and left } \\
\text { parietal) activity }\end{array}$ & [14] \\
\hline & & Leaves & Immunotoxicity & Larvae of Aedes aegypti & [44] \\
\hline \multirow[t]{2}{*}{3} & \multirow[t]{2}{*}{ Angelica glauca Edgew } & \multirow[t]{2}{*}{ Whole plant } & $\begin{array}{l}\text { Antioxidant, antimicrobial, and } \\
\text { phytotoxic }\end{array}$ & $\begin{array}{l}\text { Bacteria: Staphylococcus aureus, Bacillus subtilis, Escherichia } \\
\text { coli, and Pasteurella multocida } \\
\text { Fungi: Candida albicans, Microsporum canis, Aspergillus flavus } \\
\text { and Fusarium solani. } \\
\text { DPPH radical scavenging assay } \\
\text { Phytotoxic activity against Lemna minor }\end{array}$ & [24] \\
\hline & & & Broncho-relaxant & $\begin{array}{l}\text { Airway was induced using histamine aerosol in guinea pigs } \\
\text { and ovalbumin aerosol in albino mice. }\end{array}$ & [45] \\
\hline
\end{tabular}


Table 2. Cont.

\begin{tabular}{|c|c|c|c|c|c|}
\hline S. No. & Species & Parts & Biological activity & Model & References \\
\hline \multirow{9}{*}{4} & \multirow{9}{*}{ Angelica sinensis (Oliv.) Diels } & \multirow{9}{*}{ Root } & Anti-inflammatory & Carrageenan-induced rats & [32] \\
\hline & & & Antioxidant & DPPH, ABTS scavenging, and $\beta$-carotene bleaching assays. & [46] \\
\hline & & & Anti-inflammatory & $\begin{array}{l}\text { Carrageenan-induced rats and mechanism by plasma } \\
\text { metabolomics approach }\end{array}$ & [34] \\
\hline & & & Antibacterial & $\begin{array}{l}\text { Staphylococcus aureus, Staphylococcus chromogenes, and } \\
\text { Streptococcus uberis }\end{array}$ & [47] \\
\hline & & & Anti-inflammatory & Carrageenan-induced acute inflammation model rats & [48] \\
\hline & & & Anti-inflammatory & Lipopolysaccharide-induced inflammation rat model & {$[33,49]$} \\
\hline & & & \multirow[t]{2}{*}{ Anxiolytic } & $\begin{array}{l}\text { Elevated plus-maze, light/dark and stress-induced } \\
\text { hyperthermia tests }\end{array}$ & [50] \\
\hline & & & & Social interaction test of anxiety and the hole-board test & [51] \\
\hline & & & Repellent & Against Aedes aegypti & [35] \\
\hline 5 & Angelica koreana Maxim. & Root & Antifungal and antioxidant & $\begin{array}{l}\text { Aspergillus (A. flavus, A. fumigaus, A. niger, A. terreus and } A \text {. } \\
\text { versicolor) and Trichophyton (T. mentagrophytes, T. rubrum and } \\
\text { T. tonsurans) species } \\
\text { DPPH scavenging, nitrite inhibition, and reducing power }\end{array}$ & [36] \\
\hline \multirow{5}{*}{6} & \multirow{5}{*}{$\begin{array}{l}\text { Angelica dahurica (Fisch. Ex Hoffm.) } \\
\text { Benth. \& Hook. }\end{array}$} & \multirow{5}{*}{ Root } & $\begin{array}{l}\text { Anti-inflammatory and } \\
\text { immunomodulating properties }\end{array}$ & $\begin{array}{l}\text { Xylene-induced acute ear swelling and } \\
\text { carrageenan-induced acute paw edema in mice; } \\
\text { anti-inflammatory and immunomodulating properties in } \\
\text { Freund's complete adjuvant (FCA)-induced arthritis in rats. }\end{array}$ & [19] \\
\hline & & & $\begin{array}{l}\text { Enhance sensitivity of } \\
\text { MCF-7/ADR breast cancer cells } \\
\text { to doxorubicin }\end{array}$ & MDR human breast cancer MCF-7/ADR cells & [52] \\
\hline & & & Insecticidal & $\begin{array}{l}\text { Yellow fever mosquito, Aedes aegypti, and azalea lace bugs, } \\
\text { Stephanitis pyrioides }\end{array}$ & [20] \\
\hline & & & Antibacterial & $\begin{array}{l}\text { Staphylococcus aureus, Staphylococcus chromogenes, and } \\
\text { Streptococcus uberis }\end{array}$ & [47] \\
\hline & & & Immunotoxicity & Larvae of Aedes aegypti & [44] \\
\hline 7 & Angelica pubescentis Maxim. & Root & Antifungal and Insecticidal & $\begin{array}{l}\text { Colletotrichum acutatum, Colletotrichum fragariae, and } \\
\text { Colletotrichum gloeosporioides, Yellow fever mosquito, Aedes } \\
\text { aegypti, and azalea lace bugs, Stephanitis pyrioides }\end{array}$ & [20] \\
\hline 8 & $\begin{array}{l}\text { Angelica anomala Avé-Lall., Angelica } \\
\text { cartilagino-marginata var. distans, } \\
\text { Angelica czernevia, Angelica decursiva } \\
\text { (Miq.) Franch. \& Sav., Angelica fallax } \\
\text { H. Boissieu, Angelica japonica A. Gray }\end{array}$ & Leaves & Immunotoxicity & Larvae of Aedes aegypti & [44] \\
\hline \multirow[t]{2}{*}{9} & \multirow[t]{2}{*}{ Angelica species } & \multirow[t]{2}{*}{ Root } & $\begin{array}{l}\text { Penetration Enhancers for } \\
\text { Transdermal Administration of } \\
\text { Ibuprofen }\end{array}$ & $\begin{array}{l}\text { Therapeutic efficacy of ibuprofen with essential oil was } \\
\text { evaluated using dysmenorrheal model mice }\end{array}$ & [53] \\
\hline & & & Skin permeation of drugs & Skin permeation of ibuprofen across rat abdominal skin & [54] \\
\hline
\end{tabular}

DPPH: 1,1-diphenyl-1-picrylhydrazyl; ABTS: 2,2-azino-bis(3ethylbenzo-thiazoline-6-sulfonic acid); EEG: electroencephalographic activity; MDR: multidrug resistance. 
Kim et al. [29] determined the EO composition from the rhizomes of $A$. gigas, A. sinensis, and A. acutiloba by solvent-free solid injector method. Coumarin derivatives such as decursinol angelate $(16.83 \%)$ and decursin $(29.34 \%)$ were found to be the most abundant components, followed by lomatin $(10.25 \%)$, and marmesin $(9.33 \%)$ in A. gigas. Butylidene dihydro-phthalide, $(15.23 \%)$, butylidene phthalide (14.27\%), furfural (16\%), and camphene (10.66\%) were the main components in $A$. sinensis. Similarly, butylidene phthalide (17.82\%) and furfural (13.67\%) were registered as the major components in A. acutiloba.

Sowndhararajan et al. [14] compared the EO composition of $A$. gigas root by steam distillation and supercritical carbon dioxide extract $\left(\mathrm{SC}-\mathrm{CO}_{2}\right)$. The EO mainly composed of monoterpene hydrocarbons $(52.83 \%)$, followed by oxygenated sesquiterpenes $(25.53 \%)$. In these, $\alpha$-pinene $(28.64 \%), \beta$-eudesmol $(14.80 \%)$, nonane $(8.49 \%)$, and $\gamma$-eudesmol (5.97\%) were the major components in the EO of $A$. gigas root. However, decursin (40.13\%) and decursinol angelate $(28.44 \%)$ were detected as the most abundant components in $\mathrm{SC}-\mathrm{CO}_{2}$. $\alpha$-Pinene $(30.89 \%)$ was also the major component in the EO of $A$. gigas extracted by simultaneous steam distillation and extraction method [31]. In another study, the roots of A. gigas and A. acutiloba were collected from the field of Snyder Research and Extension Farm Rutgers University, New Jersey, and analyzed for their EO composition. The main constituents of the A. gigas root EO were ligustilide (47\%) and $\gamma$-terpinene $(14 \%)$. In the case of $A$. acutiloba root EO, $\alpha$-pinene $(32 \%)$ and nonane $(25 \%)$ were the major components [28].

Chen et al. [30] compared the volatile compositions of $A$. acutiloba roots, stems, and leaves using steam distillation and headspace solid-phase microextraction (HS-SPME). In all three parts, a total of 61 and 33 compounds were detected by SD and HS-SPME, respectively. In the steam distillation, $3 n$-butyl phthalide, $\gamma$-terpinene, $p$-cymene, and cis- $\beta$-ocimene were the main compounds. On the other hand, $\gamma$-terpinene and $p$-cymene were the main compounds in HS-SPME. Further, the authors reported that monoterpene components were found to be higher in the HS-SPME sampling method when compared with steam distillation.

In the EO of $A$. major, $\alpha$-pinene $(21.8 \%)$ and cis- $\beta$-ocimene $(30.4 \%)$ were found to be the most abundant components [55]. The main components in A. dahurica EO were $\alpha$-pinene (46.3\%), sabinene (9.3\%), myrcene (5.5\%), 1-dodecanol (5.2\%), and terpinen-4-ol (4.9\%). In regards to A. pubescentis root EO, $\alpha$-pinene $(37.6 \%)$, p-cymene $(11.6 \%)$, limonene $(8.7 \%)$, and cryptone $(6.7 \%)$ were found to be the major components [20]. Champakaew et al. (2015) found that 3-N-butylphthalide, butylidene phthalide, ligustilide, and di-iso-octyl phthalate were the main components in $A$. sinensis EO. The composition of EOs of the stem and leaves of Angelica urumiensis were studied by Mohammadi et al. [38]. In the EO from the leaves, $\alpha$-cadinol (20.2\%), hexahydrofarnesyl acetone (10.03\%), and 1-dodecanol (7.55\%) were the major components. On the other hand, $\alpha$-cadinol $(9.24 \%)$ and $\delta$-cadenine $(6.11 \%)$ were the major components in the EO from the stem. The EO compositions of $A$. pancicii were compared by GC-MS liquid injection and headspace-GC-MS modes. In total, 40 compounds were identified in the EO by GC-MS liquid injection, and 44 by HS-GC-MS. In both cases, the main components were $\beta$-phellandrene, $\alpha$-pinene, and $\alpha$-phellandrene [37]. Caryophyllene oxide (61.7\%) and $\alpha$-pinene $(67.2 \%)$ were detected as the most abundant components in EOs of A. viridiflora and A. cincta aerial parts, respectively [39].

\section{Biological Activities of Angelica Essential Oils}

\subsection{Antioxidant}

1,1-Diphenyl-2-picrylhydrazil (DPPH) and 2,2-azino-bis(3ethylbenzo-thiazoline-6-sulfonic acid (ABTS) radical scavenging activities are extensively used measures to evaluate the antioxidant potential of plant extracts or compounds. DPPH, nitrite inhibition, and reducing power were determined to assess the antioxidant activity of Angelica koreana EO and its major components. $m$-Cresol (56.12\%) showed stronger DPPH scavenging activity than EO (19.31\%) and sabinene $(4.45 \%)$ at the concentration of $16 \mathrm{mg} / \mathrm{mL}$. Additionally, sabinene exhibited the strongest reducing 
power and nitric oxide scavenging activities than the EO fraction or $m$-cresol [36]. Irshad et al. [24] reported that $A$. glauca EO exhibited good DPPH radical scavenging and peroxidation inhibition activities. Angelica seed oil showed 39\% of DPPH radical scavenging activity at the concentration of $200 \mu \mathrm{g} / \mathrm{mL}$ [40]. The antioxidant activity of $A$. sinensis was investigated by DPPH, ABTS, and beta-carotene bleaching assays. A. sinensis $\mathrm{EO}$ and coniferyl ferulate rich fractions 1 and 2 showed strong DPPH ( $\mathrm{IC}_{50}$ of 194.7, 42.4 and $15.2 \mu \mathrm{g} / \mathrm{mL}$, respectively) and ABTS (IC 50 of 98.8, 15.9 and $7.8 \mu \mathrm{g} / \mathrm{mL}$, respectively) radical scavenging activities. Further, coniferyl ferulate rich fractions 1 and 2 exhibited good $\beta$-carotene bleaching activity with $\mathrm{IC}_{50}$ values of 11.0 and $2.0 \mu \mathrm{g} / \mathrm{mL}$, respectively [46]. In another study, the DPPH radical scavenging activity of $A$. archangelica EO, $\alpha$-terpineol, phenyl ethyl alcohol, and their combination were determined. The $\mathrm{IC}_{50}$ values of $A$. archangelica $\mathrm{EO}, \alpha$-terpineol, and their EO-based combination were 1.04, 66.6, and $3.89 \mu \mathrm{L} / \mathrm{mL}$, respectively [42].

\subsection{Antimicrobial}

A. koreana $\mathrm{EO}$ and its main components, sabinene and $m$-cresol, showed antifungal activity against different species of Aspergillus and Trichophyton with minimal inhibitory concentrations (MICs) of $125-1000 \mu \mathrm{g} / \mathrm{mL}$. In addition, EO exhibited synergistic activity when combined with itraconazole [36]. The EO of A. glauca showed appreciable antimicrobial activity against selected strains of bacteria (Staphylococcus aureus, Bacillus subtilis, Escherichia coli, and Pasteurella multocida) and fungi (Candida albicans, Microsporum canis, Aspergillus flavus, and Fusarium solani). Among the bacterial strains tested, Escherichia coli and Staphylococcus aureus were the most sensitive bacteria with minimum inhibitory concentration (MIC) values of 141.3 and $159.3 \mu \mathrm{g} / \mathrm{mL}$, respectively. In regards to fungal strains, Microsporum canis was the most sensitive organism with a MIC value of $178.1 \mu \mathrm{g} / \mathrm{mL}$ [24].

The EO of $A$. archangelica root showed considerable antimicrobial activity against Clostridium difficile, Clostridium perfringens, Enterococcus faecalis, Eubacterium limosum, Peptostreptococcus anaerobius, and Candida albicans. Further, A. archangelica EO showed a weaker antimicrobial activity against the intestinal microflora such as bifidobacteria and lactobacilli. In another study, the EO showed antifungal activity against some species of the Fusarium genus, Botrytis cinerea, and Alternaria solani [22,27]. A combination of $A$. archangelica EO: Phenyl ethyl alcohol (PEA): $\alpha$-terpineol (1:1:1) inhibited the growth of Aspergillus flavus NKDW-7 (aflatoxigenic strain) and aflatoxin B1 production at 2.25 and $2.0 \mu \mathrm{L} / \mathrm{mL}$, respectively. At the concentration of $2.0 \mu \mathrm{L} / \mathrm{mL}$, the combination showed a $>90 \%$ decrease in ergosterol content in the plasma membrane of Aspergillus flavus [42].

Cavaleiro et al. [55] evaluated the antifungal activity of the EO of Angelica major and its major components, $\alpha$-pinene and cis- $\beta$-ocimene, against clinically important yeasts and molds. A. major EO exhibited a broad spectrum of antifungal activity, including all tested fungi (animal and human pathogenic species or spoilage fungi): Candida spp., C. neoformans, Aspergillus spp., and dermatophytes. $\alpha$-Pinene was more active against all of the tested fungi than cis- $\beta$-ocimene. A. sinensis and A. dahurica EOs exhibited significant antibacterial activity against three mastitis-causing pathogens: Staphylococcus aureus, Staphylococcus chromogenes, and Streptococcus uberis [47]. Tabanca et al. [20] reported that $A$. pubescentis root EO exhibited weak antifungal activity against Colletotrichum acutatum, Colletotrichum fragariae, and Colletotrichum gloeosporioides. In the case of A. dahurica root EO, there was no antifungal activity observed against tested fungal strains.

\subsection{Insecticidal}

EOs from the root of $A$. dahurica and A. pubescentis were studied as pest management prospectives. When compared with $A$. pubescentis EO, A. dahurica EO showed better biting deterrent and insecticidal activity against Aedes aegypti and Stephanitis pyrioides. In mosquito bioassays, components of $A$. dahurica EO, 1-dodecanol and 1-tridecanol, showed antibiting deterrent activity against Aedes aegypti [20]. Chung et al. [44] investigated the immunotoxicity effect of EOs from the leaves of $A$. anomala, A. cartilagino-marginata var. distans, A. czernevia, A. dahurica, A. decursiva, Angelica fallax, A. gigas, and A. japonica. Among them, the $\mathrm{EO}$ of $A$. dahurica showed a significant toxic effect against early 
fourth-stage larvae of Aedes aegypti, with a $\mathrm{LC}_{50}$ value of $43.12 \mathrm{ppm}$. In another study, out of 33 plant species tested, $A$. sinensis EO showed the best repellent activity against Aedes aegypti, with a median complete protection time of $7.0 \mathrm{~h}$ [35].

\subsection{Behavioral}

Repeated administration of nicotine can produce behavioral sensitization, and this is a good model for studying drug addiction. Zhao et al. [43] reported that the inhalation of $A$. gigas EO significantly ameliorated nicotine-induced behavioral sensitization by decreasing dopamine release in the nucleus accumbens and locomotor activity in repeated nicotine-induced rats. Pathak et al. [41] found that the EO of the A. archangelica root exhibited antiseizure activity against electrically and chemically-induced seizures in mice. Chen et al. [50] investigated the anxiolytic activity of Angelica EO in a mice model. The results revealed that the EO of Angelica exhibited considerable anxiolytic-like effects at the concentration of $30.0 \mathrm{mg} / \mathrm{kg}$ (orally), as measured in the elevated plus-maze, the light/dark, and the stress-induced hyperthermia tests. In addition, Angelica EO significantly improved the behavioral performances in the social interaction test of anxiety and the hole-board test of exploration and locomotor activity in rats [51]. Sharma et al. [45] reported that the EO of A. glauca exhibited broncho-relaxant activity against histamine and ovalbumin-induced bronchoconstriction in guinea pigs by decreasing absolute blood eosinophil count, serum levels of immunoglobulin E, and the number of eosinophils and neutrophils in bronchoalveolar lavage fluid. Sowndhararajan et al. [14] investigated the effect of inhalation of $\mathrm{EO}$ of $A$. gigas root on electroencephalographic activity in humans. The results revealed that absolute low beta significantly increased at left temporal and left parietal region during the inhalation of the EO of A. gigas root, and these changes may contribute to the enhancement of language learning abilities in humans.

\subsection{Anti-Inflammatory}

Zhang et al. [32] used the metabonomics based on GC-MS to study the possible anti-inflammatory mechanisms of $\mathrm{EO}$ of $A$. sinensis in rats with acute inflammation. In the carrageenan-injected rats, treatment with the $\mathrm{EO}$ of $A$. sinensis significantly restored the levels of prostaglandin E2, histamine, and 5-hydroxytryptamine in the inflammatory fluid, similar to the normal group. GC-MS analysis identified 14 metabolite biomarkers detected in the inflammatory fluid. Zhong et al. [48] evaluated the anti-inflammatory effect of EOs obtained from processed products of $A$. sinensis. For this purpose, EOs from stir-fried $A$. sinensis, fried $A$. sinensis with alcohol, cooked $A$. sinensis with soil, and fried $A$. sinensis with sesame oil were applied to intervene the carrageenan-induced acute inflammation of the model rats. The results showed that the EOs of $A$. sinensis significantly inhibited the release of prostaglandin E2, histamine, 5-hydroxytryptamine, and tumor necrosis factor- $\alpha$. Furthermore, A. sinensis exhibited an anti-inflammatory effect against the lipopolysaccharide (LPS)-induced inflammation rat model by regulating the Krebs cycle, enhancing the glucose content, and restoring the fatty acid metabolism [33].

$\mathrm{Li}$ et al. [49] investigated the effects of $A$. sinensis EO on the LPS-induced acute inflammation rat model. A. sinensis EO exhibited anti-inflammatory and liver protection effects by inhibiting the secretion of the pro-inflammatory cytokines (tumor necrosis factor- $\alpha$, interleukin- $1 \beta$, and interleukin- 6 ), the inflammatory mediators (histamine, 5-hydroxytryptamine, prostaglandin E2, and nitric oxide), the inflammation-related enzymes (inducible nitric oxide synthase and cyclooxygenase 2), as well as promoting the production of the anti-inflammatory cytokines interleukin-10. Wang et al. [19] reported that the EO of $A$. dahurica (at $100 \mathrm{mg} / \mathrm{kg}$ ) showed anti-inflammatory activity against xylene-induced ear swelling and carrageenan-induced paw edema in a mice model. In addition, the EO significantly alleviated Freund's complete adjuvant-induced arthritis in rats by improving hind paw swelling and reducing the serum levels of nitric oxide, tumor necrosis factor- $\alpha$, prostaglandin E2, and serum nitric oxide synthase activity. 


\subsection{Skin Permeation Enhancer}

It is well known that EOs can reversibly overcome the stratum corneum barrier to improve the skin permeation of drugs. Chen et al. [53] studied the penetration enhancement effect of five EOs (clove, Angelica, Chuanxiong, Cyperus, and cinnamon) on the transdermal drug delivery of ibuprofen using dysmenorrheal model mice. Among five EOs tested, Chuanxiong and Angelica oils effectively enhanced the transdermal drug delivery of ibuprofen. In another study, turpentine, Angelica, Chuanxiong, Cyperus, cinnamon, and clove oils (at $3 \% w / v)$ were evaluated for the potential to enhance the skin penetration of ibuprofen in rats. When compared with azone, the tested EOs had significantly higher penetration enhancement effect and lower skin irritation potential. The results revealed that EOs can enhance the skin permeation of ibuprofen mainly by disturbing the stratum corneum lipids [54].

\section{Conclusions}

EOs have been isolated from different plant parts of Angelica species. The most abundant components in the EOs were $\alpha$-pinene, $\beta$-pinene, $\alpha$-phellandrene, $\beta$-phellandrene, $\delta$ - 3 -carene, sabinene, $\gamma$-terpinene, limonene, $p$-cymene, ligustilide, butylidene phthalide, $\alpha$-cadinol, and $\beta$-eudesmol. Based on the previous reports, the EOs from different Angelica species exhibit appreciable antioxidant, antimicrobial, insecticidal and anti-inflammatory activities. In addition, EOs significantly enhance behavioral performances and promote the skin permeation of drugs. Among the different Angelica species, $A$. archangelica, $A$. sinensis, and $A$. dahurica were the most studied plant species in relation to the biological activities of EOs. This review will offer a scientific basis for future studies in relation to biological activities of EO-bearing plants.

Acknowledgments: This work was supported by Korea Institute of Planning and Evaluation for Technology in Food, Agriculture, Forestry and Fisheries (IPET) through High Value-added Food Technology Development Program, funded by Ministry of Agriculture, Food and Rural Affairs (MAFRA) (317044-03) and the research grant from Kangwon National University, Chuncheon, Republic of Korea.

Author Contributions: Kandhasamy Sowndhararajan wrote the manuscript; Ponnuvel Deepa and Minju Kim conducted the literature review; Se Jin Park and Songmun Kim corrected and revised the manuscript.

Conflicts of Interest: The authors declare no conflict of interest.

\section{References}

1. Clardy, J.; Walsh, C. Lessons from natural molecules. Nature 2004, 432, 829-837. [CrossRef]

2. Raut, J.S.; Karuppayil, S.M. A status review on the medicinal properties of essential oils. Ind. Crops Prod. 2014, 62, 250-264. [CrossRef]

3. Bakkali, F.; Averbeck, S.; Averbeck, D.; Idaomar, M. Biological effects of essential oils-A review. Food Chem. Toxicol. 2008, 46, 446-475. [CrossRef] [PubMed]

4. Carson, C.F.; Hammer, K.A. Chemistry and bioactivity of essential oils. In Lipids and Essential Oils as Antimicrobial Agents; Thormar, H., Ed.; John Wiley \& Sons: Oxford, UK, 2011; pp. 203-238.

5. Mohammadhosseini, M.; Mahdavi, B.; Shahnama, M. Chemical composition of essential oils from aerial parts of Ferula gummosa (Apiaceae) in Jajarm Region, Iran using traditional hydrodistillation and solvent-free microwave extraction methods: A comparative approach. J. Essent. Oil Bear. Plants 2015, 18, 1321-1328. [CrossRef]

6. Mohammadhosseini, M.; Sarker, S.D.; Akbarzadeh, A. Chemical composition of the essential oils and extracts of Achillea species and their biological activities: A review. J. Ethnopharmacol. 2017, 199, 257-315.

7. Mohammadhosseini, M. Chemical composition of the essential oils and volatile fractions from flowers, stems and roots of Salvia multicaulis Vahl. by using MAHD, SFME and HS-SPME methods. J. Essent. Oil Bear. Plants 2015, 18, 1360-1371.

8. Nekoei, M.; Mohammadhosseini, M. Chemical compositions of the essential oils from the aerial parts of Achillea wilhelmsii using traditional hydrodistillation, microwave assisted hydrodistillation and solvent-free microwave extraction methods: Comparison with the volatile compounds obtained by headspace solid phase microextraction. J. Essent. Oil Bear. Plants 2016, 19, 59-75. 
9. Feng, T.; Downie, S.R.; Yu, Y.; Zhang, X.; Chen, W.; He, X.; Liu, S. Molecular systematics of Angelica and allied genera (Apiaceae) from the Hengduan Mountains of China based on nrDNA ITS sequences: Phylogenetic affinities and biogeographic implications. J. Plant Res. 2009, 122, 403-414. [CrossRef]

10. Chen, J.T.; Li, H.Z.; Wang, D.; Yang, C.R.; Liu, Y. Determination of sucrose in Radix Angelicae sinensis by HPLC-ELSD. Mod. Chin. Med. 2008, 10, 19-20.

11. Wei, W.L.; Zeng, R.; Gu, C.M.; Qu, Y.; Huang, L.F. Angelica sinensis in China-A review of botanical profile, ethnopharmacology, phytochemistry and chemical analysis. J. Ethnopharmacol. 2016, 190, 116-141. [CrossRef] [PubMed]

12. Hook, I.L. Danggui to Angelica sinensis root: Are potential benefits to European women lost in translation? A review. J. Ethnopharmacol. 2014, 152, 1-13. [CrossRef] [PubMed]

13. Sowndhararajan, K.; Kim, S. Neuroprotective and cognitive enhancement potentials of Angelica gigas Nakai root: A review. Sci. Pharm. 2017, 85, 21. [CrossRef] [PubMed]

14. Sowndhararajan, K.; Seo, M.; Kim, M.; Kim, H.; Kim, S. Effect of essential oil and supercritical carbon dioxide extract from the root of Angelica gigas on human EEG activity. Complement. Ther. Clin. Pract. 2017, 28, 161-168. [CrossRef] [PubMed]

15. Hatano, R.; Takano, F.; Fushiya, S.; Michimata, M.; Tanaka, T.; Kazama, I.; Suzuki, M.; Matsubara, M. Water-soluble extracts from Angelica acutiloba Kitagawa enhance hematopoiesis by activating immature erythroid cells in mice with 5-fluorouracil-induced anemia. Exp. Hematol. 2004, 32, 918-924. [CrossRef] [PubMed]

16. Pasqua, G.; Monacelli, B.; Silvestrini, A. Accumulation of essential oils in relation to root differentiation in Angelica archangelica L. Eur. J. Histochem. 2003, 47, 87-90. [CrossRef] [PubMed]

17. Chauhan, R.S.; Nautiyal, M.C.; Cecotti, R.; Mella, M.; Tava, A. Variation in the essential oil composition of Angelica archangelica from three different altitudes in Western Himalaya, India. Ind. Crops Prod. 2016, 94, 401-404. [CrossRef]

18. Agnihotri, V.K.; Thappa, R.K.; Meena, B.; Kapahi, B.K.; Saxena, R.K.; Qazi, G.N.; Agarwal, S.G. Essential oil composition of aerial parts of Angelica glauca growing wild in North-West Himalaya (India). Phytochemistry 2004, 65, 2411-2413. [CrossRef] [PubMed]

19. Wang, C.; Sun, J.; Li, H.; Yang, X.; Liu, H.; Chen, J. In vivo anti-inflammatory activities of the essential oil from Radix Angelicae dahuricae. J. Nat. Med. 2016, 70, 563-570. [CrossRef] [PubMed]

20. Tabanca, N.; Gao, Z.; Demirci, B.; Techen, N.; Wedge, D.E.; Ali, A.; Sampson, B.J.; Werle, C.; Bernier, U.R.; Khan, I.A.; et al. Molecular and phytochemical investigation of Angelica dahurica and Angelica pubescentis essential oils and their biological activity against Aedes aegypti, Stephanitis pyrioides, and Colletotrichum species. J. Agric. Food Chem. 2014, 62, 8848-8857. [CrossRef] [PubMed]

21. Nivinskiene, O.; Butkiene, R.; Mockute, D. Chemical composition of seed (fruit) essential oils of Angelica archangelica L. growing wild in Lithuania. Chemija 2005, 16, 51-54.

22. Fraternale, D.; Flamini, G.; Ricci, D. Essential oil composition and antimicrobial activity of Angelica archangelica L. (Apiaceae) roots. J. Med. Food 2014, 17, 1043-1047. [CrossRef] [PubMed]

23. Nivinskiene, O.; Butkiene, R.; Mockute, D. The chemical composition of the essential oil of Angelica archangelica L. roots growing wild in Lithuania. J. Essent. Oil Res. 2005, 17, 373-377. [CrossRef]

24. Irshad, M.; Rehman, H.U.; Shahid, M.; Aziz, S.; Ghous, T. Antioxidant, antimicrobial and phytotoxic activities of essential oil of Angelica glauca. Asian J. Chem. 2011, 23, 1947-1951.

25. Purohit, V.K.; Andola, H.C.; Haider, S.Z.; Tiwari, D.; Bahuguna, Y.M.; Gairola, K.C.; Arunachalam, K. Essential Oil Constituents of Angelica glauca Edgew. Roots: An Endangered Species from Uttarakhand Himalaya (India). Natl. Acad. Sci. Lett. 2015, 38, 445-447. [CrossRef]

26. Sigurdsson, S.; Ogmundsdottir, H.M.; Gudbjarnason, S. The cytotoxic effect of two chemotypes of essential oils from the fruits of Angelica archangelica L. Anticancer Res. 2005, 25, 1877-1880. [PubMed]

27. Fraternale, D.; Flamini, G.; Ricci, D. Essential oil composition of Angelica archangelica L. (Apiaceae) roots and its antifungal activity against plant pathogenic fungi. Plant Biosyst. 2016, 150, 558-563. [CrossRef]

28. Park, C.H.; Juliani, H.R.; Park, H.W.; Yu, H.S.; Simon, J.E. Comparison of essential oil composition between Angelica gigas and Angelica acutiloba. Korean J. Plant Resour. 2003, 6, 183-187.

29. Kim, M.R.; Abd El-Aty, A.M.; Kim, I.S.; Shim, J.H. Determination of volatile flavor components in danggui cultivars by solvent free injection and hydrodistillation followed by gas chromatographic-mass spectrometric analysis. J. Chromatogr. A 2006, 1116, 259-264. [CrossRef] [PubMed] 
30. Chen, H.C.; Tsaia, Y., Jr.; Linb, L.Y.; Wu, C.S.; Tai, S.P.; Chen, Y.C.; Chiang, H.M. Volatile compounds from roots, stems and leaves of Angelica acutiloba growing in Taiwan. Nat. Prod. Commun. 2014, 9, 583-586. [PubMed]

31. Seo, H.Y.; Yang, S.H.; Shim, S.L.; No, K.M.; Park, K.S.; Song, K.D.; Kim, K.S. Volatile organic compounds of Angelica gigas Nakai, Korean medicinal herb. Nat. Prod. Res. 2007, 21, 265-273. [CrossRef] [PubMed]

32. Zhang, W.Q.; Hua, Y.L.; Zhang, M.; Ji, P.; Li, J.X.; Zhang, L.; Li, P.L.; Wei, Y.M. Metabonomic analysis of the anti-inflammatory effects of volatile oils of Angelica sinensis on rat model of acute inflammation. Biomed. Chromatogr. 2015, 29, 902-910. [CrossRef] [PubMed]

33. Hua, Y.L.; Ji, P.; Xue, Z.Y.; Wei, Y.M. Construction and analysis of correlation networks based on gas chromatography-mass spectrometry metabonomics data for lipopolysaccharide-induced inflammation and intervention with volatile oil from Angelica sinensis in rats. Mol. Biosyst. 2015, 11, 3174-3187. [CrossRef] [PubMed]

34. Yao, W.; Zhang, L.; Hua, Y.; Ji, P.; Li, P.; Li, J.; Zhong, L.; Zhao, H.; Wei, Y. The investigation of anti-inflammatory activity of volatile oil of Angelica sinensis by plasma metabolomics approach. Int. Immunopharmacol. 2015, 29, 269-277. [CrossRef] [PubMed]

35. Champakaew, D.; Junkum, A.; Chaithong, U.; Jitpakdi, A.; Riyong, D.; Sanghong, R.; Intirach, J.; Muangmoon, R.; Chansang, A.; Tuetun, B.; et al. Angelica sinensis (Umbelliferae) with proven repellent properties against Aedes aegypti, the primary dengue fever vector in Thailand. Parasitol. Res. 2015, 114, 2187-2198. [CrossRef] [PubMed]

36. Roh, J.; Shin, S. Antifungal and antioxidant activities of the essential oil from Angelica koreana Nakai. Evid. Based Complement. Altern. Med. 2014, 2014, 398503. [CrossRef] [PubMed]

37. Simonović, S.R.; Stankov-Jovanović, V.P.; Mitić, V.D.; Ilić, M.D.; Petrović, G.M.; Stojanović, G.S. Chemical composition of Angelica pancicii essential oil determined by liquid and headspace GC-MS techniques. Nat. Prod. Commun. 2014, 9, 271-272. [PubMed]

38. Mohammadi, M.; Yousefi, M.; Habibi, Z. Essential oils from stem and leaves of Angelica urumiensis (Mozaffarian) from Iran. Nat. Prod. Res. 2010, 24, 1347-1351. [CrossRef] [PubMed]

39. Suleimen, E.M.; Iskakova, Z.B.; Dudkin, R.V.; Gorovoi, P.G.; Wang, M.; Khan, I.; Ross, S.A.; Martins, C.H.G. Composition and biological activity of essential oils from East-Asian species Angelica viridiflora, A. cincta, and Coelopleurum gmelinii. Chem. Nat. Compd. 2014, 50, 1136-1139. [CrossRef]

40. Wei, A.; Shibamoto, T. Antioxidant activities and volatile constituents of various essential oils. J. Agric. Food Chem. 2007, 55, 1737-1742. [CrossRef] [PubMed]

41. Pathak, S.; Wanjari, M.M.; Jain, S.K.; Tripathi, M. Evaluation of antiseizure activity of essential oil from roots of Angelica archangelica Linn. in mice. Indian J. Pharm. Sci. 2010, 72, 371-375. [PubMed]

42. Prakash, B.; Singh, P.; Goni, R.; Raina, A.K.; Dubey, N.K. Efficacy of Angelica archangelica essential oil, phenyl ethyl alcohol and $\alpha$-terpineol against isolated molds from walnut and their antiaflatoxigenic and antioxidant activity. J. Food Sci. Technol. 2015, 52, 2220-2228. [CrossRef] [PubMed]

43. Zhao, R.J.; Koo, B.S.; Kim, G.W.; Jang, E.Y.; Lee, J.R.; Kim, M.R.; Kim, S.C.; Kwon, Y.K.; Kim, K.J.; Huh, T.L.; et al. The essential oil from Angelica gigas NAKAI suppresses nicotine sensitization. Biol. Pharm. Bull. 2005, 28, 2323-2326. [CrossRef] [PubMed]

44. Chung, I.M.; Kim, E.H.; Lee, J.H.; Lee, Y.C.; Moon, H.I. Immunotoxicity activity from various essential oils of Angelica genus from South Korea against Aedes aegypti L. Immunopharmacol. Immunotoxicol. 2012, 34, 42-45. [CrossRef] [PubMed]

45. Sharma, S.; Rasal, V.P.; Patil, P.A.; Joshi, R.K. Effect of Angelica glauca essential oil on allergic airway changes induced by histamine and ovalbumin in experimental animals. Indian J. Pharmacol. 2017, 49, 55-59. [PubMed]

46. Li, S.Y.; Yu, Y.; Li, S.P. Identification of antioxidants in essential oil of radix Angelicae sinensis using HPLC coupled with DAD-MS and ABTS-based assay. J. Agric. Food Chem. 2007, 55, 3358-3362. [CrossRef] [PubMed]

47. Mullen, K.A.; Lee, A.R.; Lyman, R.L.; Mason, S.E.; Washburn, S.P.; Anderson, K.L. Short communication: An in vitro assessment of the antibacterial activity of plant-derived oils. J. Dairy Sci. 2014, 97, 5587-5591. [CrossRef] [PubMed]

48. Zhong, L.J.; Hua, Y.L.; Ji, P.; Yao, W.L.; Zhang, W.Q.; Li, J.; Wei, Y.M. Evaluation of the anti-inflammatory effects of volatile oils from processed products of Angelica sinensis radix by GC-MS-based metabolomics. J. Ethnopharmacol. 2016, 191, 195-205. [CrossRef] [PubMed] 
49. Li, J.; Hua, Y.; Ji, P.; Yao, W.; Zhao, H.; Zhong, L.; Wei, Y. Effects of volatile oils of Angelica sinensis on an acute inflammation rat model. Pharm. Biol. 2016, 54, 1881-1890. [CrossRef] [PubMed]

50. Chen, S.W.; Min, L.; Li, W.J.; Kong, W.X.; Li, J.F.; Zhang, Y.J. The effects of Angelica essential oil in three murine tests of anxiety. Pharmacol. Biochem. Behav. 2004, 79, 377-382. [PubMed]

51. Min, L.; Chen, S.W.; Li, W.J.; Wang, R.; Li, Y.L.; Wang, W.J.; Mi, X.J. The effects of Angelica essential oil in social interaction and hole-board tests. Pharmacol. Biochem. Behav. 2005, 81, 838-842. [CrossRef] [PubMed]

52. Wu, M.; Li, T.; Chen, L.; Peng, S.; Liao, W.; Bai, R.; Zhao, X.; Yang, H.; Wu, C.; Zeng, H.; et al. Essential oils from Inula japonica and Angelicae dahuricae enhance sensitivity of MCF-7/ADR breast cancer cells to doxorubicin via multiple mechanisms. J. Ethnopharmacol. 2016, 180, 18-27. [CrossRef] [PubMed]

53. Chen, J.; Jiang, Q.D.; Wu, Y.M.; Liu, P.; Yao, J.H.; Lu, Q.; Zhang, H.; Duan, J.A. Potential of essential oils as penetration enhancers for transdermal administration of ibuprofen to treat dysmenorrhoea. Molecules 2015, 20, 18219-18236. [CrossRef] [PubMed]

54. Jiang, Q.; Wu, Y.; Zhang, H.; Liu, P.; Yao, J.; Yao, P.; Chen, J.; Duan, J. Development of essential oils as skin permeation enhancers: Penetration enhancement effect and mechanism of action. Pharm. Biol. 2017, 55, 1592-1600. [CrossRef] [PubMed]

55. Cavaleiro, C.; Salgueiro, L.; Gonçalves, M.J.; Hrimpeng, K.; Pinto, J.; Pinto, E. Antifungal activity of the essential oil of Angelica major against Candida, Cryptococcus, Aspergillus and dermatophyte species. J. Nat. Med. 2015, 69, 241-248. [CrossRef] [PubMed]

(C) 2017 by the authors. Licensee MDPI, Basel, Switzerland. This article is an open access article distributed under the terms and conditions of the Creative Commons Attribution (CC BY) license (http://creativecommons.org/licenses/by/4.0/). 